\title{
The Impact of Integrated Maneuver Guidance Information on UAS Pilots Performing the Detect and Avoid Task
}

\author{
Presented To: \\ Human Factors and Ergonomics Society \\ International Annual Meeting 2015
}

Conrad Rorie (Conrad.Rorie@NASA.GOV) San Jose State University Lisa Fern (Lisa.Fern@NASA.GOV) The Ohio State University

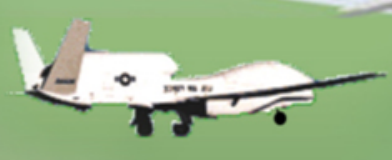




\section{Background}

- UAS integration into the National Airspace System

- In order to comply with 14CFR section 91.113, pilots must be able to 'see and avoid' nearby traffic that would otherwise create a collision hazard

- Therefore, a Detect and Avoid (DAA) system must be developed to compensate for pilots' situated in ground control station (GCS)

- DAA System must provide information that supports pilots' ability to:

1. Detect potential threats to well clear

2. Determine appropriate resolutions

3. Execute maneuvers using the GCS's command and control interface 


\section{Background}

- Precise information requirements have not been determined, although studies have started to investigate:

- Friedman-Berg, Rein \& Racine, 2014

- Determined a minimum amount of information (intruder state \& trend information, and alerting) in a low-fidelity simulation

- Draper, Pack, Darrah \& Moultan, 2014

- Found additional information (in form of DAA guidance) improved pilot performance and was rated as subjectively necessary by pilots

- Fern, et al., 2015

- Examined impact of traffic display location and DAA guidance tools on pilots' performance and subjective experience 


\section{Background}

- Fern et al. (2015)

- Found that an integrated traffic display (i.e., collocated with the GCS navigation and control interfaces) that included DAA guidance tools led to:

- Faster pilot response times

- Pilots able to implement their 'edits' through the GCS interface more quickly to get the aircraft maneuvering

- Higher subjective ratings

- Was rated as the best display in supporting pilots' ability to maintain well clear

- Fewer and less severe losses of well clear (Santiago et al. (2015))

- However:

- The integrated, advanced display had multiple DAA guidance tools, limiting researchers' ability to localize the source of the improved pilot response times (and lower rates of loss of well clear) 


\section{Background}

- Purpose of current study:

- Decompose the integrated, advanced display from Fern et al. (2015) to better understand its results

- Determine which, if any, display features were most responsible for faster pilot response times 


\section{Method}

\section{- Participants}

- 9 active UAS pilots $(M=46$ years of age)

- Experience:

- Military UAS (combat \& non-combat) = avg. 1182 hours

- Civilian UAS = avg. 153 hours

- 1 retired air traffic controller experienced in simulated airspace served as confederate

- 2 general aviation pilots to serve as "pseudopilots," controlling the simulated man traffic in the area 


\section{Method}

- Simulation Environment

- Ground Control Station

- Vigilant Spirit Control Station (VSCS; Air Force Research Lab)

- Tactical Situation Display (TSD; traffic information \& control interfaces)

- Out-the-window view (synthetic nose camera view with heads-up display overlay)

- Status panels (telemetry, chat window, electronic checklist)

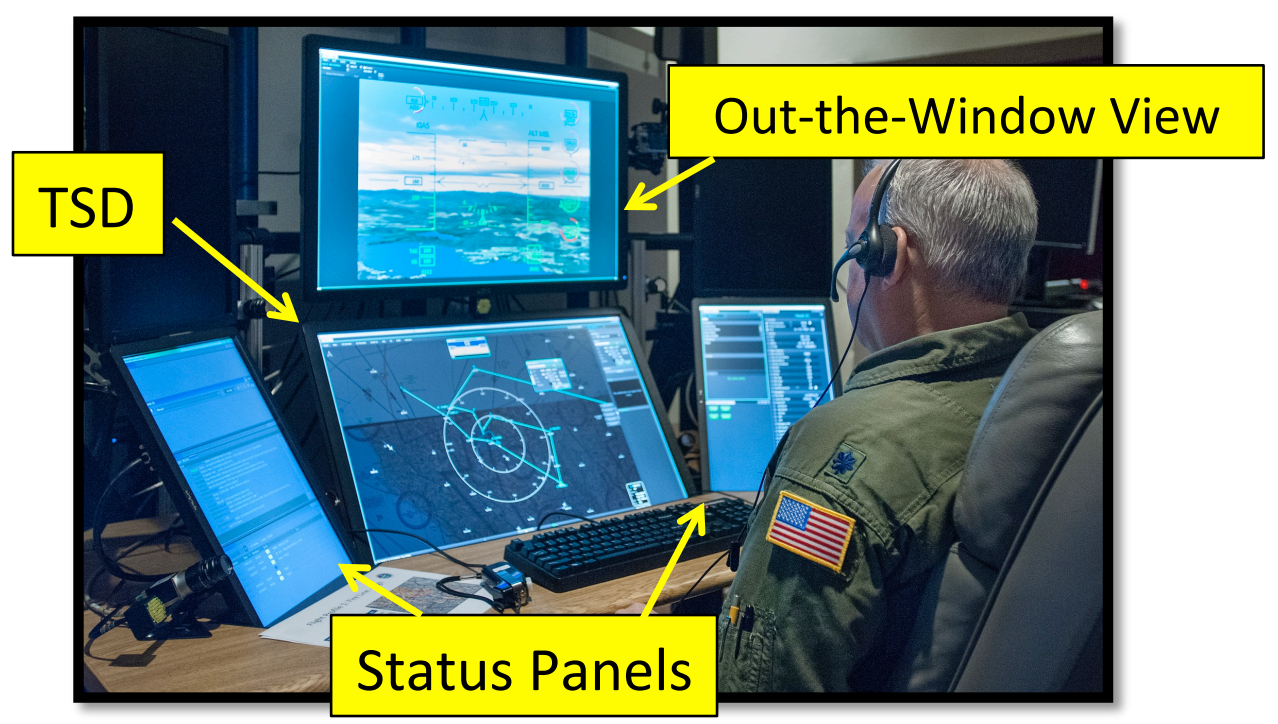




\section{Method}

- Simulation Environment

- Traffic and Airspace Simulation

- Multi Aircraft Control Station (MACS)

- Controller Display

» Provided confederate ATC with experimental sector (Oakland Center, ZOA 40/41)

- Consisted of Class A and Class E airspace

- Wide variety of aircraft types

- Traffic Generator

» Injected scripted manned aircraft into experimental sector

» Designed to emulate realistic traffic patterns and flows

- Pseudo-pilot Display

» Pilot stations allowed 2 confederate pilots to manage all simulated manned aircraft

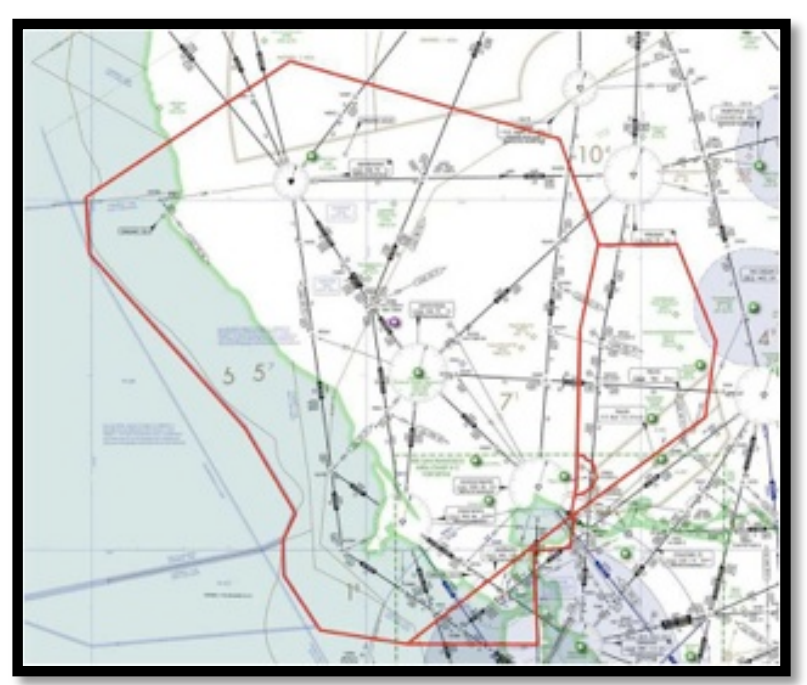




\section{Method}

- Simulation Environment

- DAA Algorithm

- Java Architecture for DAA Modeling and Extensibility (JADEM; Santiago et al., submitted)

- Compared intruder's current state to ownship's trajectory to determine if a 'loss of well clear' would occur

» Threat levels assigned according to this determination

- Contained Autoresolver-AD, which supported DAA guidance tools

\begin{tabular}{|c|c|c|c|c|}
\hline Alert Level & Icon & HMD at CPA & ZTHR & Time to CPA \\
\hline Collision Avoidance & & $<0.8 \mathrm{NM}$ & $<400 \mathrm{FT}$ & $<40$ secs \\
\hline Predicted CA Alert & $<$ & $<0.8 \mathrm{NM}$ & $<400 \mathrm{FT}$ & $<80$ secs \\
\hline Self Separation & & $<1.2 \mathrm{NM}$ & $<900 \mathrm{FT}$ & $<80$ secs \\
\hline Preventative & $<$ & $<2 \mathrm{NM}$ & $<900 \mathrm{FT}$ & $<80 \mathrm{secs}$ \\
\hline Proximal & & $>2 \mathrm{NM}$ & $>900 \mathrm{FT}$ & $\mathrm{N} / \mathrm{A}$ \\
\hline
\end{tabular}




\section{Method}

- Experimental Design

- Display Configuration

- D1: Information Only

- D2: Information + Vector Planner Tool

- D3: Information + Auto Resolver

- D4: Information + Vector Planner Tool + Auto Resolver 


\section{Method}

- Experimental Design

- Display Configuration

- D1: Information Only

- Intruder state and trend information

» Callsign

") Position \& Directionality

» Absolute and Relative Altitude

" Groundspeed

॥ Vertical Trend and Rate

» Horizontal Predictor (30sec dead reckoning)

- Alerting

॥Corresponding to status as threat to well clear 


\section{Method}

- Experimental Design

- Display Configuration

- D1: Information Only

- D2: Information + Vector Planner

- All information from D1

- Vector Planner Tool

"Allowed pilots to propose a heading or altitude prior to uploading the change

"Would reflect predicted safety level of probed maneuvers

» Integrated with Auto Pilot interface 


\section{Method}

- Experimental Design

- Display Configuration

- D1: Information Only

- D2: Information + Vector Planner

- D3: Information + Auto Resolutions

- All information from D1

- Auto Resolutions

»A single maneuver recommendation provided to pilot at onset of a Preventive, Corrective or Warning Alert

- Integrated with Auto Pilot interface 


\section{Method}

- Experimental Design

- Display Configuration

- D1: Information Only

- D2: Information + Vector Planner

- D3: Information + Auto Resolutions

- D4: Information + Vector Planner + Auto Resolutions

- All information from D1

- Vector Planner Tool (from D2) \& Auto Resolutions (from D3) also included

» Roughly same as the 'Integrated, Advanced' suite in Fern et al. (2015), which was the highest performer 


\section{Displays}

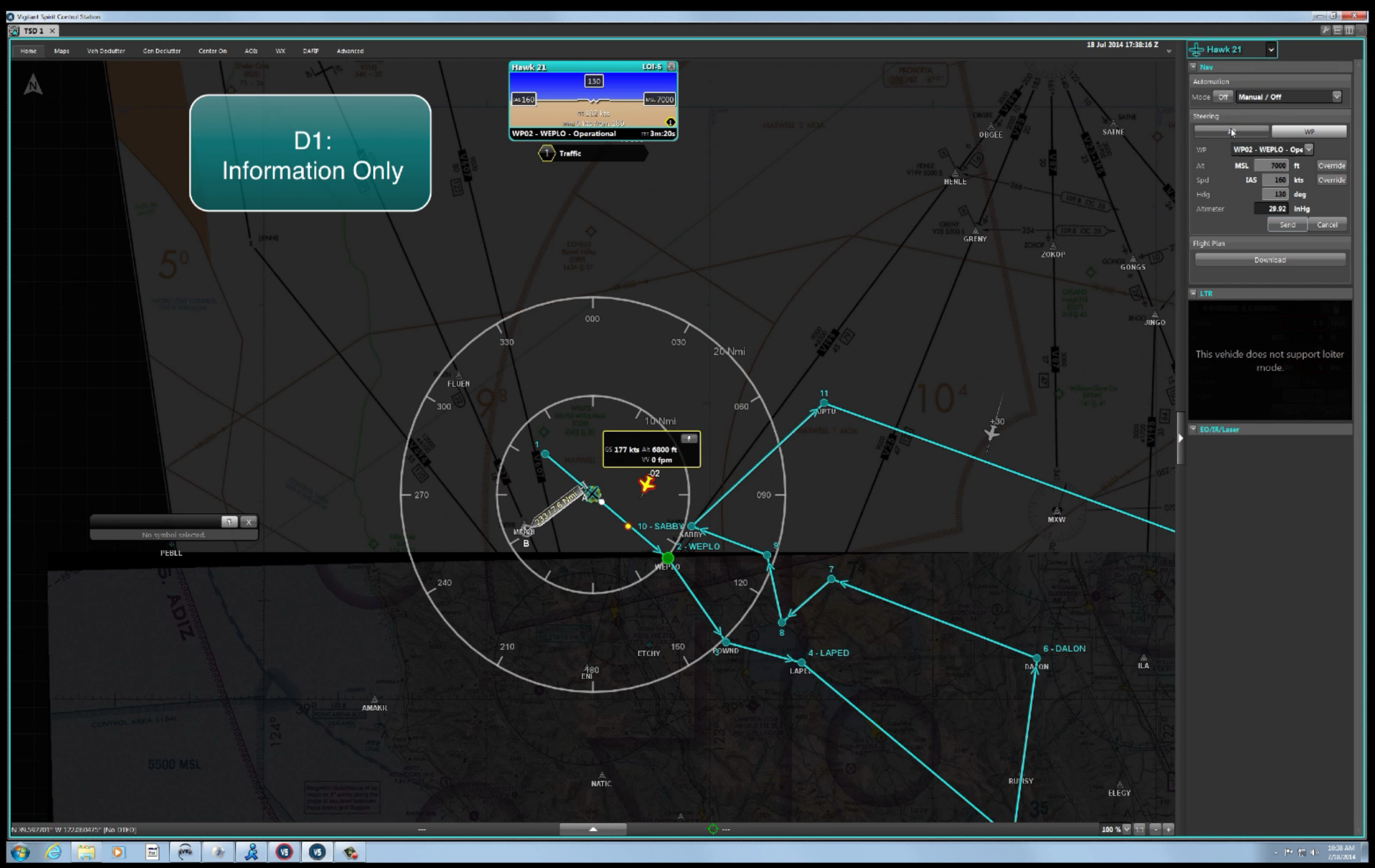


Display Conditions
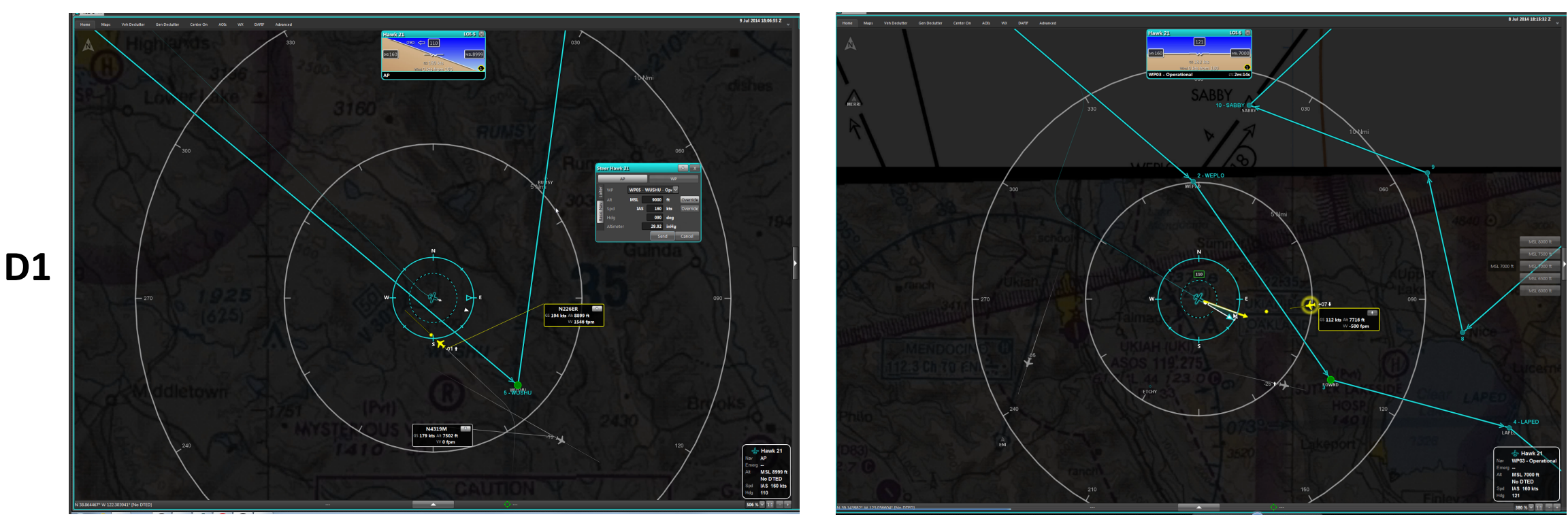

D2
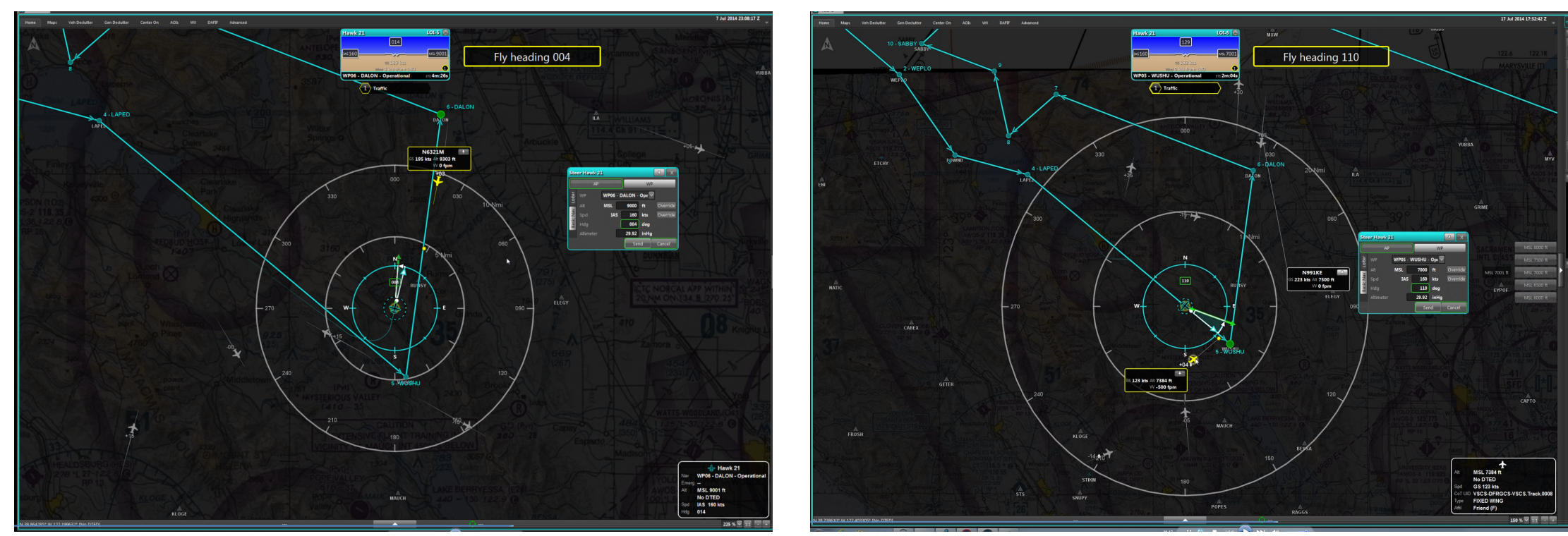

D4 


\section{Method}

- Primary Tasks:

1. Maintain well clear from other aircraft

- Coordinate with ATC regarding any deviations from filed route and altitude

- Monitor traffic display to determine when maneuvers may be necessary

2. Fly pre-filed path as much as practical

- Only responsible for navigating the aircraft (IFR)

- Simulated performance specs of MQ-9 Reaper

- Requires interaction with the GCS and coordination with ATC

- Secondary tasks:

1. 'Chat' directed

- "Mission" Radio Frequency changes

- Radial and Distance check

- Nominal information requests (e.g., fuel level remaining)

2. System Alerts

- Generator Failure (checklist)

- Tanker Header Overpressure (checklist)

- Annunciator checks 


\section{Method}

- Scenarios

- Pilots flew 2 different routes

- Both within Class E

- Each had a dedicated scripted traffic scenario

-40 min route

- 8 encounters with ownship intended to lose well clear

- Pilots free to maneuver as appropriate

- Training

- Initial training just on operating simulated vehicle through Vigilant Spirit Control Station interfaces

- Dedicated training for each display configuration immediately prior to experimental trials

- Order of presentation counterbalanced between participants 


\section{Method}

- Measures

- Pilot response time metrics adapted from Fern et al. (2015)

- Provide insight into pilots' ability to respond to threats of well clear in timely manner

- Pilots' response times help define the the minimum operational performance standards (MOPS) for expected pilot and system performance

- Referenced in human models during fast time simulation

- Metrics derived using a pilot-DAA interaction timeline

\begin{tabular}{|l|l|}
\hline Stage & Description \\
\hline$T_{0}$ & Self separation alert appears on display \\
\hline$T_{1}$ & Pilot notifies ATC of desire to maneuver \\
\hline$T_{2}$ & ATC approves pilot request \\
\hline$T_{3}$ & Pilot initiates an edit using VSCS control interfaces \\
\hline$T_{4 a}$ & Pilots uploads first maneuver to aircraft \\
\hline$T_{4 b}$ & Pilots uploads final maneuver to aircraft \\
\hline
\end{tabular}




\section{Stages of Pilot DAA Task}

- Notification Time (T1-T0)

- Calculated as the time between the appearance of an alert and the beginning of the pilot's transmission to ATC

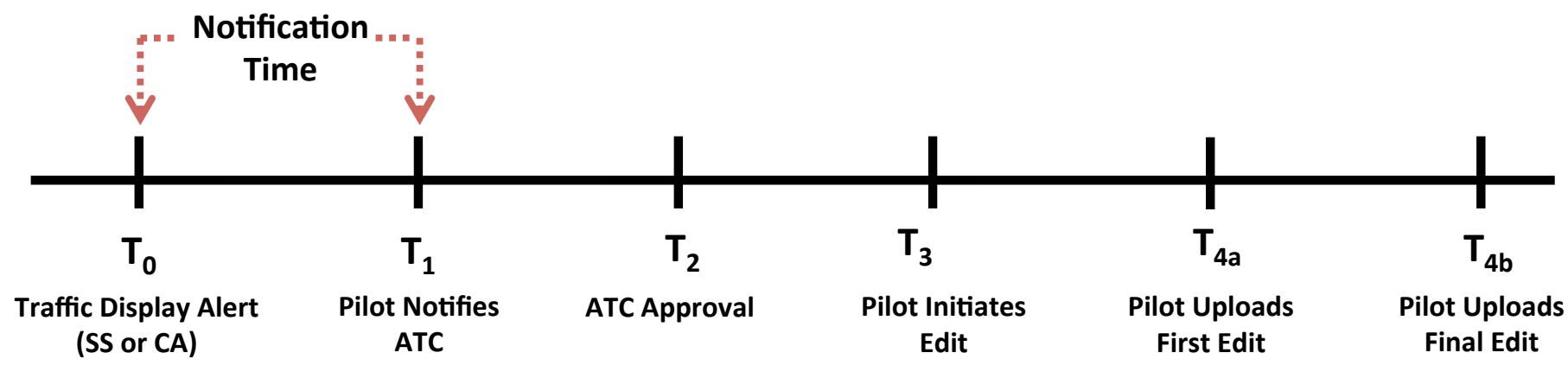




\section{Stages of Pilot DAA Task}

- Notification Time

- Initial Response Time (T3-T0)

- Calculated as the difference between the appearance of an alert and the pilot's first explicit interaction with the vehicle control interfaces

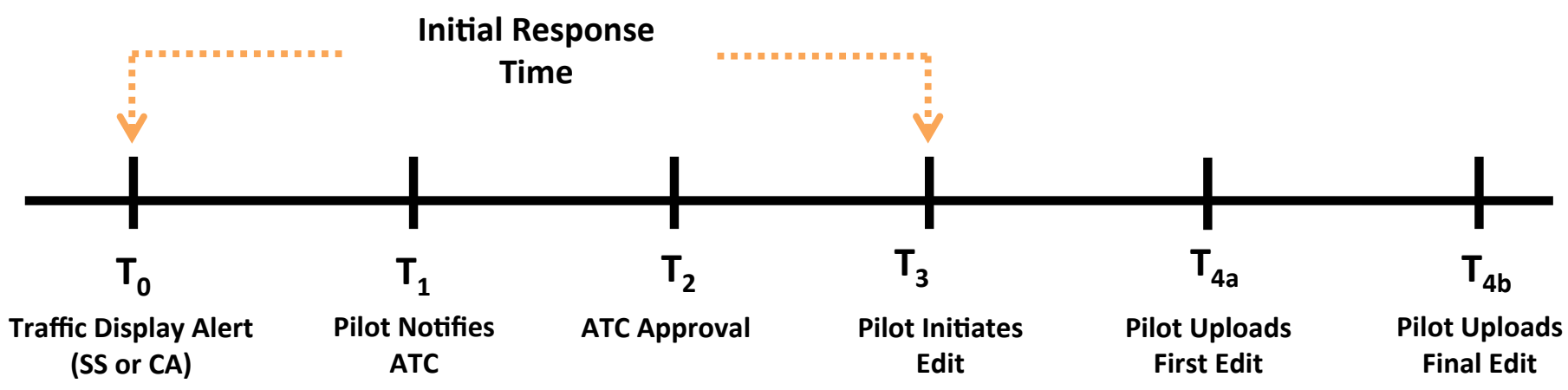




\section{Stages of Pilot DAA Task}

- Notification Time

- Initial Response Time

- Initial Edit Time (T4a - T3)

- Calculated as the time between the pilot's first explicit interaction with the vehicle control interfaces and the pilot's first upload to the aircraft

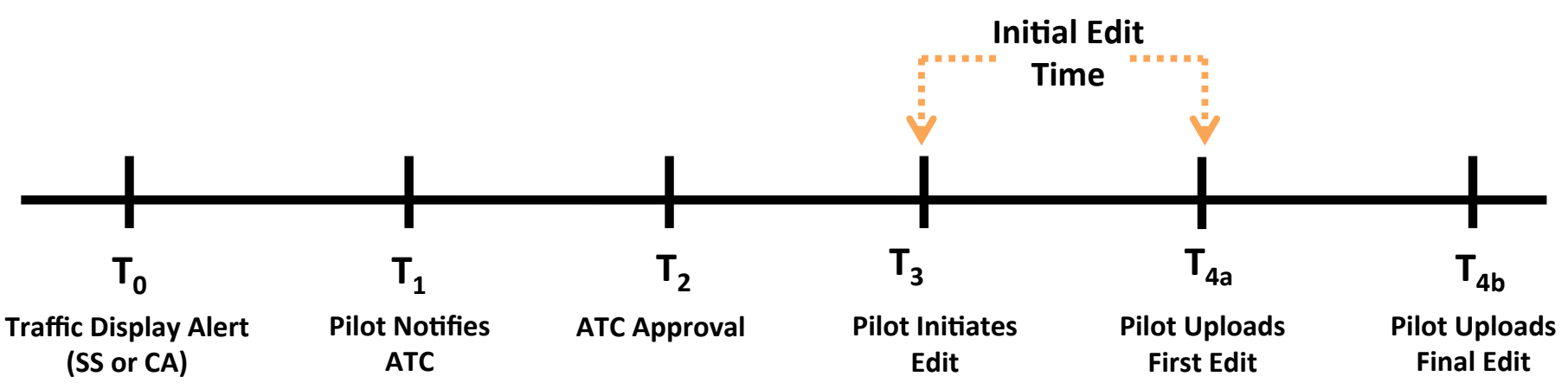




\section{Stages of Pilot DAA Task}

- Notification Time

- Initial Response Time

- Initial Edit Time

- $\quad$ Total Edit Time (T4b - T3)

- Calculated as the time between the pilot's first explicit interaction with the vehicle control interfaces and the pilot's final upload to the aircraft

- Identical to Initial Edit Time when only one upload is made

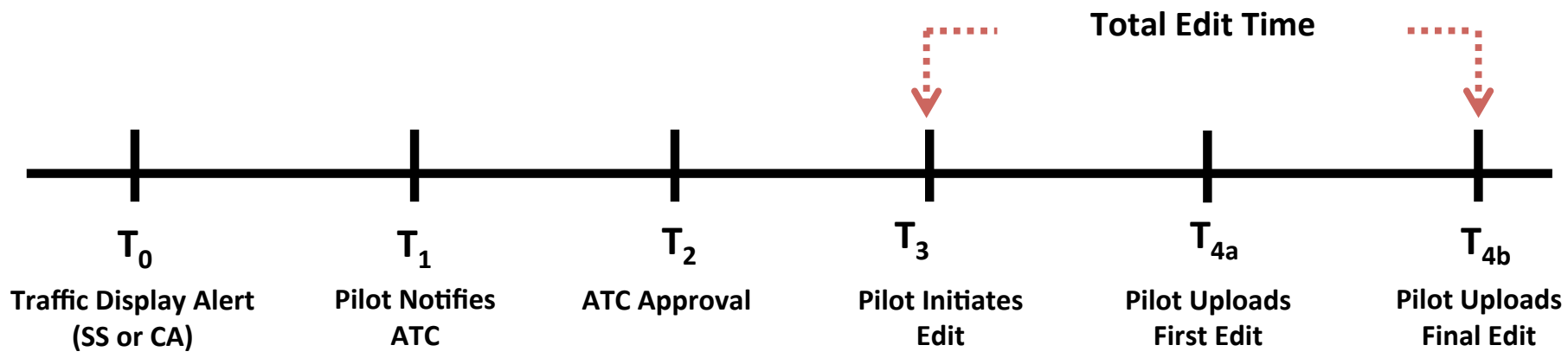




\section{Stages of Pilot DAA Task}

- Notification Time

- Initial Response Time

- Initial Edit Time

- Total Edit Time

- Total Response Time (T4b-T0)

- Calculated as the time between the onset of a self separation alert and the final upload to the aircraft

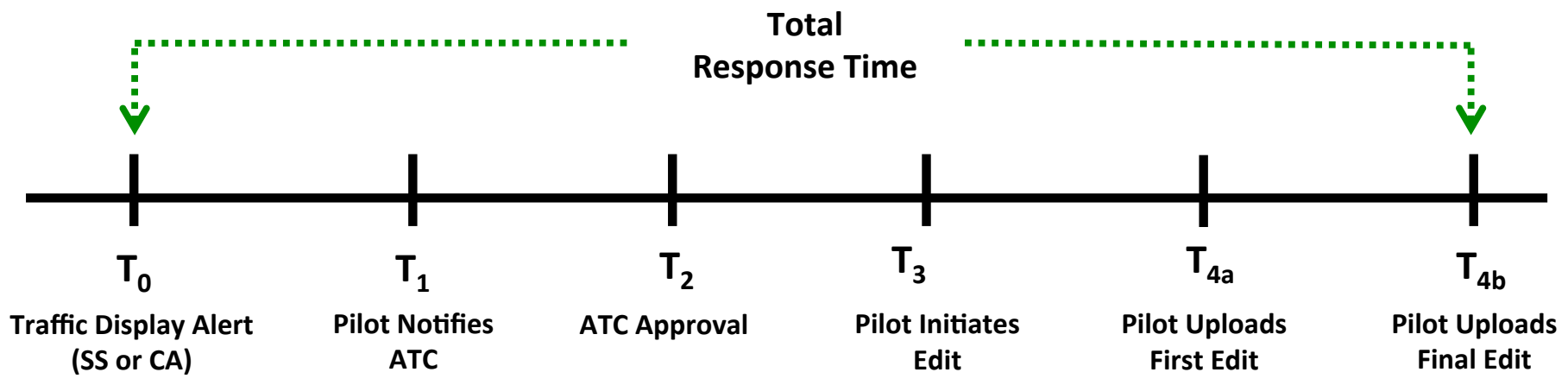




\section{RESULTS}

RESPONSE TIMES BY DISPLAY CONFIGURATION WITHIN-SUBJECTS ANALYSIS OF VARIANCE 


\section{Results}

- Notification Time

- No significant main effect of display configuration ( $p>.05)$

- Initial Response Time

- No significant main effect of display configuration ( $p>.05)$

\begin{tabular}{|l|l|l|l|l|}
\multicolumn{1}{|c|}{ Metric } & \multicolumn{1}{c|}{ D1 } & \multicolumn{1}{c|}{ D2 } & \multicolumn{1}{c|}{ D3 } & \multicolumn{1}{c|}{ D4 } \\
\hline Notification Time & 13.68 & 13.61 & 12.01 & 14.89 \\
& $(1.63)$ & $(2.45)$ & $(2.40)$ & $(2.82)$ \\
\hline Initial Response Time & 7.83 & 7.14 & 9.45 & 6.87 \\
& $(1.49)$ & $(1.14)$ & $(2.28)$ & $(0.94)$
\end{tabular}




\section{Results}

- Initial Edit Time

- Significant main effect $(p<.001)$

- D3 \& D4 had significantly shorter initial edit times than D1 \& D2

- Total Edit Time

- Significant main effect $(p<.05)$

- D4 had significantly shorter total edit times than D2

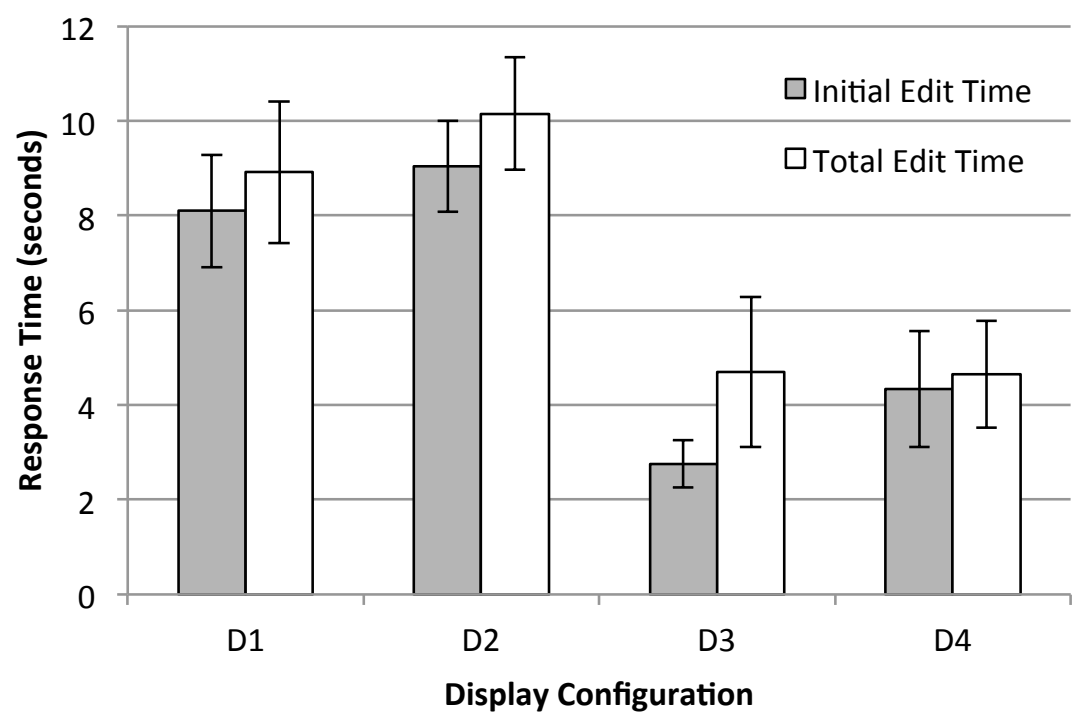




\section{Results}

- Total Response Times

- Approached significance $(p=.10)$

- D4 led to the shortest total response times, while D2 led to longest

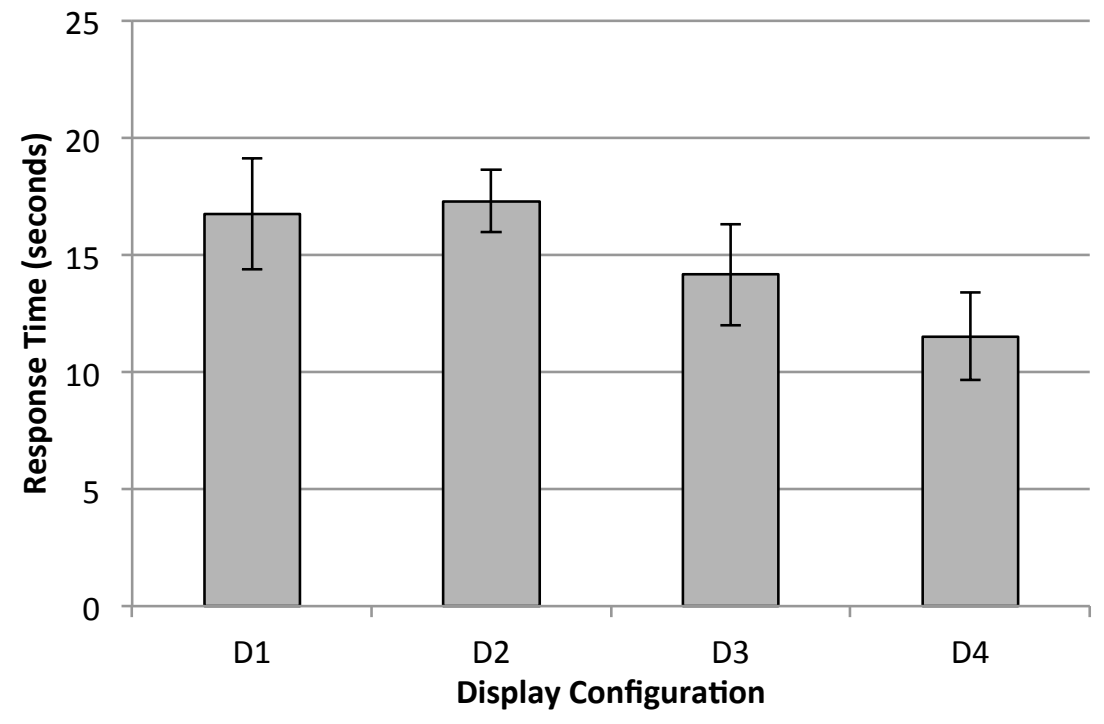




\section{Discussion}

- Study was an attempt to better understand findings of Fern et al. (2015)

- Found pilots had fastest response times when using an Integrated, Advanced display

- Current findings suggest the benefits were due to the Auto Resolver tool

- Present in D3 and D4 configuration which:

- Cut edit times from $46 \%$ to $70 \%$

- Cut total response times by $33 \%$

- No difference seen for notification and initiate times 


\section{Discussion}

- Source of improvements in Auto Resolver conditions likely due to 2 things:

1. Directive maneuver guidance

- By providing a single solution Auto Resolver reduced the amount of time it took pilots to decide on an appropriate course of action

- In D1, pilots had to determine their own resolution entirely, while in D2 pilots could use the Vector Planner Tool to decide on necessary maneuver

» Resulted in D2 corresponding to longer edit times than D1

2. Coupling of Auto Resolutions with Auto Pilot control interfaces in Vigilant Spirit

- By auto-populating the auto pilot control interfaces, pilots were not required to make any 'edits' in the case that they were comfortable with the automation's recommendation

- Pilots complied with Auto Resolution's suggestion $70 \%$ of the time 


\section{Discussion}

- Pilots found to respond to alerts, overall, in a timely manner

- Total response times were reduced when pilots had Auto Resolver available, however the times were not significantly different

- Relative to Fern et al. (2015) pilots responded more quickly

- Across all conditions, total response times were reduced by $50 \%$

- Likely due to improved alerting criteria and simplified training

- Emphasizes the impact ground control station interfaces can have on pilot response times

- By integrating tools with auto pilot interfaces we saw greater benefits than may otherwise be seen 


\section{Discussion}

- Limitations

- Small sample size $(n=9)$ reduced ability to find significant differences

- Response times do not directly equate to safer system performance

- Pilots can respond quickly but ineffectively

- Loss of well clear rates and severity of losses of well clear are critical

- Santiago and Mueller (submitted) found that D4 resulted in the lowest rate of, and least severe, losses of well clear

» D1 and D2 had the highest rates

- Future Research

- Look into de-coupling the guidance from the GCS auto-pilot control interfaces

- The coupling is not likely to be a 'minimum requirement' and therefore may not provide realistic response times

- Simulate different forms of DAA guidance

- "Banding" displays provide constant, explicit feedback

- Replicate experiment with different GCS interfaces

- Results therefore not entirely generalizable - GCS control interfaces vary wildly between platforms 